\title{
LES ORGANISATIONS HYBRIDES
}

\author{
Chantal Hervieux \\ Saint Mary's University \\ Jean-Mathieu Fallu \\ HEC Montréal-École des Hautes Études commerciales \\ Marie-France Turcotte \\ Université du Québec à Montréal
}

\begin{abstract}
In this article, we study hybrid organizations, namely those practicing social entrepreneurship and those practicing durable entrepreneurship. These organizations do not choose between the contradictory requirements fostered by the objectives that they pursue, be they double objectives (social and economic) or triple (social, economic and environmental). Rather, they choose to accept the tensions inherent in the pursuit of contradictory objectives. This study is based on a cluster analysis of 244 small- and medium-sized enterprises (SMEs). It identifies and distinguishes between three main types of organization, commercial SMEs, social entrepreneurship SMEs and sustainable SMEs. These types of SMEs, even though they resemble each other in their structural characteristics (size, age, revenue), differ in terms of the actualization of their entrepreneurial and market orientations.

\section{RÉSUMÉ}

Dans cet article, nous étudions les organisations hybrides, c'est-à-dire celles de l'entrepreneuriat social et de l'entrepreneuriat durable. Ces organisations, plutôt que de choisir entre les exigences contradictoires posées par les objectifs doubles (social et économique) ou triples (social, économique et environnemental) qu'elles s'efforcent d'atteindre, choisissent d'accepter les tensions créées par la poursuite d'objectifs contradictoires. L'étude permet, à partir d'une analyse de regroupement réalisée auprès de 244 PME, d'identifier et de distinguer trois principaux types d'organisation, soit les PME commerciales, les PME d'entreprenariat social et les PME d'entrepreneuriat durable. En outre, ces types de PME, dont les caractéristiques structurelles (taille, âge, revenus) ne sont pas différentes, diffèrent en ce qui concerne l'actualisation de l'orientation entrepreneuriale et de l'orientation envers le marché.
\end{abstract}

KEYWORDS / MOTS CLÉS : Social entrepreneurship; Durable entrepreneurship; Hybrid organizations; Entrepreneurial orientation; Market orientation / Entrepreneuriat social; Entrepreneuriat durable; Organisations hybrides; Orientation entrepreneuriale; Orientation vers le marché 


\section{Hervieux, Fallu, \& Turcotte (2016)}

\section{INTRODUCTION}

Notre article se concentre sur deux formes d'organisations hybrides qui, plutôt que de choisir entre les exigences contradictoires des objectifs doubles et triples qu'elles s'efforcent d'atteindre, choisissent d'accepter les tensions ainsi créées. Ces organisations sont des hybrides sociales (organisations d'entrepreneuriat social) ainsi que des hybrides durables (organisations d'entrepreneuriat durable).

Les organisations d'entrepreneuriat social (ES) peuvent revêtir diverses formes; elles peuvent être des organisations sans but lucratif, à but lucratif, ou un mélange des deux (Nicholls, 2006). Ces organisations cependant partagent toutes deux points communs : 1) l'entreprise accorde la priorité à la création de valeur sociale et 2) elle s'efforce de s'acquitter de cette mission au moyen de nouveaux modes d'entrepreneuriat et d'innovation (Nicholls, 2006). Les organisations d'ES sont par conséquent des organisations hybrides, c'est-àdire "des modes d'organisation novateurs qui allient de façon unique des éléments organisationnels et structurels propres aux entreprises à but lucratif et non lucratif ... ainsi que la recherche simultanée de création de richesse publique et privée » (Florin et Schmidt, 2011, p. 166). On peut considérer que les dirigeants de ces organisations acceptent la tension engendrée par la poursuite d'objectifs opposés (Battilana et al., 2012). La première tension, liée aux objectifs sociaux, oriente les décisions vers la redistribution des profits, alors que la seconde, liée aux objectifs commerciaux, oriente plutôt les décisions vers l'efficacité et la concurrence (Pache et Santos, 2013).

D'autres organisations hybrides vont plus loin dans la mesure où elles intègrent également des considérations environnementales. Dans ces organisations hybrides, les valeurs sociales, environnementales et économiques sont étroitement liées (Wilson et Post, 2013). Ces organisations intègrent donc les différentes dimensions du développement durable et acceptent de vivre avec de telles tensions entre les objectifs poursuivis.

Les organisations hybrides amalgament plusieurs identités qui sont parfois incompatibles à première vue (Albert et Whetten, 1985). Cette incompatibilité doit être résolue et les acteurs organisationnels explorent pour ce faire des stratégies leur permettant de gérer la complexité (Smith et Lewis, 2011). Notre recherche porte sur cet aspect. Nous allons étudier les orientations stratégiques des organisations hybrides leur apportant la flexibilité nécessaire pour faire face à cette complexité. La notion d'orientation stratégique se rapporte aux mécanismes de sélection favorisés par la direction qui visent à assurer la cohérence entre les intentions stratégiques et les activités poursuivies (Atuahene-Gima \& Ko, 2001).

Les organisations hybrides sont complexes et « nécessite(nt) une équipe de direction en mesure ... de faire face à l'ambiguitté et de laisser la place à l'incertitude » (Smith et Lewis, 2011, p. 396). La gestion de l'hybridité dans les organisations nécessite aussi l'adoption d'orientations stratégiques favorables à sa complexité; elle nécessite des capacités dynamiques. Deux de ces capacités dynamiques liées aux organisations hybrides sociales ont fait l'objet de discussions : l'orientation entrepreneuriale et l'orientation vers le marché (Zahra, 2008). Ces deux orientations stratégiques procurent à l'entreprise le dynamisme nécessaire en favorisant la proactivité, la prise de risques, l'innovation et l'autonomie (orientation entrepreneuriale) tout en tenant compte des informations provenant du marché (orientation marché).

Nous présentons dans un premier temps l'entrepreneuriat social (ES) et l'entrepreneuriat durable, puis deux capacités dynamiques, l'orientation entrepreneuriale (OE) et l'orientation vers le marché (OM). Nous poursuivons en expliquant pourquoi ces capacités sont importantes pour les organisations hybrides sociales et 


\section{Hervieux, Fallu, \& Turcotte (2016)}

durables. Nous exposons par la suite la méthodologie de la recherche, les analyses et les résultats, avant de conclure.

À l'heure actuelle, les données empiriques sur les organisations hybrides sont limitées. Cet article permettra de définir les capacités dynamiques des organisations hybrides sociales et durables. Pour ce faire, nous classifierons, à l'aide d'une analyse de regroupement (cluster analysis), un échantillon de 244 organisations (PME) en fonction des dimensions du développement durable (sociale, environnementale et commerciale). Dans un deuxième temps, nous approfondirons l'analyse de ces organisations en fonction des ressources et des orientations dynamiques jugées favorables pour gagner et conserver un avantage concurrentiel durable (orientation entrepreneuriale et orientation vers le marché) (Zahra, 2008).

\section{ENTREPRENEURIAT SOCIAL : L’ENTREPRISE HYBRIDE SOCIALE}

II existe une variété de concepts se rapportant aux organisations étudiées dans notre recherche, dont deux sont importants à distinguer : les concepts d'entreprise sociale et d'entrepreneuriat social. Brouard et Larivet (2010) soulignent que l'entrepreneuriat social est un sous-concept de celui d'entreprise sociale. Ceux-ci soutiennent que les entreprises sociales ne sont pas nécessairement entrepreneuriales, ces deux concepts étant liés par les orientations sociales des organisations s'y rattachant, mais ne représentant pas nécessairement le même ensemble d'organisations : seules les entreprises sociales entrepreneuriales seraient en effet comprises dans le sous-ensemble des organisations de l'entrepreneuriat social. Nous avons donc retenu le concept d'organisations d'entrepreneuriat social (ES), car c'est sur ce sous-ensemble que porte notre recherche.

Les organisations d'ES visent à créer une valeur économique et sociale (Austin, Stevenson et Wei Skillern, 2006). Ces organisations, tout comme les organismes de bienfaisance et sans but lucratif, accordent la priorité aux objectifs sociaux (Peredo et McLean, 2006) et emploient des méthodes fondées sur le marché pour obtenir les ressources nécessaires en vue d'atteindre ces objectifs (Lasprogata et Cotten, 2003; Mair et Marti, 2006). Ainsi, les organisations d'entrepreneuriat social « allient l'objectif social traditionnellement associé au secteur sans but lucratif à la rationalité économique et aux approches fondées sur le marché traditionnellement associées aux entreprises à but lucratif » (Wilson et Post, 2013, p. 715).

Les motivations des entreprises de se tourner vers le mode organisationnel de l'entrepreneuriat social découleraient de la réduction des fonds provenant de sources traditionnelles et de la recrudescence de la concurrence pour ces fonds entre les organisations à vocation sociale et les organisations commerciales (Weerarwardena, McDonald et Mort, 2010). C'est donc cette augmentation de la concurrence et la diminution des fonds disponibles qui ont poussé les organisations sociales à adopter des stratégies entrepreneuriales (Zahra, Gedajlovic, Neubaum, et Shulman, 2009), ainsi que les méthodes d'affaires des entreprises commerciales (Eikenberry et Kluver, 2004). Bien que les organisations d'entrepreneuriat social puissent être à but lucratif, elles diffèrent des entreprises commerciales dans la mesure où leur principal objectif est ancré dans leur mission sociale. Par conséquent, les organisations d'entrepreneuriat social constituent « un phénomène organisationnel distinct, car elles semblent combiner délibérément et explicitement, dès leur création, les caractéristiques des deux secteurs » (Wilson et Post, 2013, p. 716). L'encadré 1 ci-dessous présente un exemple d'ES. 


\section{Encadré 1 : Exemple d'initiative d'entrepreneuriat social}

\section{Le Robin des Bois (http://robindesbois.ca/)}

\section{Mission commerciale}

Le Robin des Bois est un restaurant situé à Montréal. Par la vente de ses produits ainsi que les revenus de son restaurant, cette organisation sans but lucratif vise la redistribution auprès d'organisations impliquées dans leur communauté. L'entreprise verse donc tous ses profits aux organisations suivantes : Jeunesse au Soleil, Le Chaînon, Santropol Roulant, Le Refuge.

\section{Mission sociale}

En plus de soutenir les organismes mentionnés plus haut, le Robin des Bois vise à « encourager le bénévolat créatif, l'engagement et le don de soi; sensibiliser les gens aux besoins des plus démunis de notre communauté ». La fondatrice, Judy Servay, souhaite rendre le bénévolat plus accessible et amener ainsi les gens à participer dans leur communauté.

Les entrepreneurs sociaux sont définis comme des agents de changement qui appliquent des méthodes novatrices pour apporter une solution à des problèmes sociaux persistants (Zahra et al., 2009). Le domaine de l'entrepreneuriat social constitue un « domaine d'études extrêmement intersectoriel et un secteur d'activité (englobant les secteurs sans but lucratif, à but lucratif et public) qui tire parti de la créativité et de l'innovation (particularités du domaine de l'entrepreneuriat traditionnel) tout en étant spécifiquement axé sur le changement social (Perrini, 2006) » (Wilson et Post, 2013, p. 716).

Pour certains, les organisations d'ES existeraient lorsqu'elles allient une occasion d'affaires à l'innovation sociale, créant ainsi une valeur sociale dans le processus de recherche d'une occasion d'affaires rentable (Wilson et Post, 2013). Cela n'implique pas pour autant que les organisations d'ES doivent nécessairement être des entreprises à but lucratif (Nicholls, 2006). Dans notre article, la conception mise de l'avant sur les organisations d'ES englobe toutes les organisations qui combinent des objectifs sociaux et commerciaux, tout en accordant la priorité aux activités sociales plutôt que commerciales, qu'elles soient à but lucratif ou non. L'entrepreneuriat social est donc lié à l'économie sociale, mais diffère de cette dernière qui regroupe des organisations démocratiques et dont l'activité « peut être vue comme une action collective axée autour de trois dimensions-sociale, économique et politique » (Brouard et al., 2010, p. 51). L'entrepreneuriat social n'est ni nécessairement démocratique, ni politique (Brouard et al., 2010). Or il a nécessairement un objectif principal qui est social et il utilise des moyens commerciaux ou d'affaires pour atteindre ses objectifs (Hervieux et al., 2010). Certains ont critiqué cette association d'objectifs contradictoires, soulignant la possibilité que la logique du marché éloigne les organisations d'ES de leurs objectifs sociaux. Pourtant, des recherches s'étant penchées sur le discours des acteurs institutionnels (Ruebottom, 2013) indiquent que les acteurs des organisations d'ES tiennent un discours qui met l'accent sur l'aspect positif de cette tension entre logiques marchande et sociale (Ruebottom, 2013). Les organisations d'ES ont tendance à accepter les tensions créées par les doubles identités inhérentes à cette forme organisationnelle. Les réponses stratégiques mises en place par ces organisations mèneraient à l'adoption de façons de faire entrepreneuriales (Sharir et Lerner, 2006), d'une position orientée vers l'innovation (Weerarwardena et al., 2010), ainsi que d'une orientation vers le marché (Nicholls, 2006; Nicholls et Cho, 2006). 


\section{L'ENTREPRENEURIAT DURABLE : ORGANISATIONS HYBRIDES DURABLES}

Tandis que les organisations d'ES accordent la priorité aux objectifs sociaux par rapport aux objectifs commerciaux, les organisations d'entrepreneuriat durable se distinguent dans la mesure où elles aspirent à être des entreprises commerciales durables. La vision des entrepreneurs sociaux consiste à atténuer un problème social (Austin et al., 2006; Veda et Kidwell, 2007). Les entrepreneurs durables « se concentrent (plutôt) sur une idée commerciale qui équilibre les répercussions économiques, sociales et environnementales de leurs activités » (Spence, Gherib et Biwole, 2010, p. 340). Ces entreprises recherchent des solutions « mutuellement gagnantes » qui remettent profondément en question des hypothèses enracinées selon lesquelles le développement durable n'est possible qu'en sacrifiant la rentabilité économique (Cohen et Winn, 2007). Comme le proposent Spence et al. (2010), les entrepreneurs qui dirigent des entreprises durables doivent avoir une vision claire de l'orientation de leur entreprise et cette orientation doit inclure le développement durable. Ils figureraient parmi les premiers à trouver des occasions d'affaires durables et à y donner suite avant leurs concurrents, étant donné qu'ils estiment que cette stratégie proactive peut constituer une source de profit (Shapero, 1975) et/ou qu'elle peut créer de la valeur et préserver la richesse pour les générations futures (Biondi, Iraldo et Meredith, 2002; Longo, Mura et Bonoli, 2005).

Ces organisations hybrides adoptent une orientation entrepreneuriale qui conçoit l'innovation comme ayant le potentiel de résoudre des défis sociaux et environnementaux, puisque « l'exploitation du potentiel novateur de l'entrepreneuriat pour relever les défis environnementaux au moyen de solutions d'affaires novatrices permettra de réaliser des gains réels » (Cohen et Winn, 2007, p. 30). Contrairement à l'entrepreneuriat social où la plupart considèrent que les profits sont seulement pertinents dans la mesure où ils aident l'organisation à réaliser sa mission sociale (Dees, 1998), l'entrepreneuriat durable est par définition à but lucratif. Par conséquent, « les innovations nécessaires proviendront vraisemblablement des entrepreneurs qui sont en mesure de trouver des occasions, d'obtenir des rentes de l'entrepreneur tout en relevant simultanément des défis environnementaux et sociaux, progressant ainsi vers les triples résultats » (Cohen et Winn, 2007, p. 30) (voir encadré 2).

Les organisations hybrides sociales (entrepreneuriat social) et les organisations hybrides durables adoptent toutes deux des orientations entrepreneuriales et marchandes qui leur apportent des compétences utiles à la réconciliation de leurs identités multiples. Nous présentons ces deux orientations dans la prochaine section.

\section{Encadré 2 : Exemple d'initiative d'entrepreneuriat durable}

Café Rico (http://www.caferico.qc.ca/\#le_cafe_rico)

\section{Mission commerciale}

Petit café de quartier, Café Rico est un restaurant et torréfacteur de café engagé dans la vente de produits équitables et biologiques. L'entreprise se décrit en fait comme une entreprise à « $100 \%$ équitable et biologique ».

\section{Mission sociale}

Café Rico pratique ce qu'il nomme « un commerce équitable engagé » et poursuit plusieurs objectifs sociaux : travailler en partenariat avec les producteurs du Sud en favorisant la redistribution des profits, faire du restaurant un lieu de rencontre culturel et social, favoriser la création de liens de solidarité auprès des producteurs québécois. 


\section{ORIENTATION ENTREPRENEURIALE ET ORIENTATION VERS LE MARCHE}

Lumpkin et Dess (1996) distinguent l'entrepreneuriat de l'orientation entrepreneuriale (OE). II s'agit d'une distinction similaire à celle faite entre le contenu de la stratégie et le processus. L'entrepreneuriat serait une nouvelle entrée (par exemple, la pénétration de nouveaux marchés, la création de nouvelles entreprises ou d'initiatives au sein de l'entreprise); l'orientation entrepreneuriale serait le processus qui mène à une nouvelle entrée. L'orientation entrepreneuriale est donc un positionnement stratégique (Covin et Slevin, 1989) lié à la perspective du choix stratégique. L'OE n'est pas en soi l'entrepreneuriat, mais plutôt « les processus, pratiques et activités de prise de décision» (Lumpkin et Dess, 1996, p. 136) qui mènent à des initiatives entrepreneuriales (Lumpkin et Dess, 1996).

L'orientation entrepreneuriale (OE) a été conçue comme une capacité (Zahra, 2008; Bhuian, Menguc et Bell, 2005), une culture (Baker et Sinkula, 2009), une tendance ou une prédisposition (Matsuno, Mentzer et Özsomer, 2002). Ces différentes conceptions mènent à envisager l'OE comme un ensemble de capacités dynamiques résidant dans les processus organisationnels, la culture d'entreprise et les routines (Zahra, 2008) et permettant de rechercher et d'exploiter des occasions de nouvelles entrées. L'OE est aussi un mécanisme de sélection qui vise à soutenir et à promouvoir les actes d'entrepreneuriat. L'OE est donc un " mécanisme d'apprentissage social de sélection [...] qui détermine la façon dont les membres de l'organisation traitent l'information » (Atuahene-Gima et Ko, 2001, p. 55) et qui encourage la prise de risque, la proactivité et l'innovation. Ces trois dimensions sont les plus courantes dans la recherche sur l'OE (Covin et Wales, 2012). Nous choisissons d'étudier ici aussi l'autonomie, une dimension de l'entrepreneuriat proposée par Lumpkin et Dess (1996), et vise privilégier l'action entrepreneuriale au sein de l'entreprise.

L'orientation entrepreneuriale est une orientation comportementale visant à encourager la prise de risque, un comportement proactif et l'ouverture envers l'innovation (Covin et Slevin, 1989), ainsi que l'autonomie nécessaire aux acteurs organisationnels pour que ceux-ci puissent adopter de tels comportements (Lumpkin et Dess, 1996). L'ouverture envers l'innovation reflète une volonté de faire autrement que ce qui est fait habituellement, le comportement proactif vise à anticiper les besoins futurs et la prise de risques reflète la volonté d'affecter des ressources à l'initiative entrepreneuriale (Lumpkin et Dess, 1996). Quant à l'autonomie, elle renvoie à la liberté accordée aux équipes et aux individus pour que ceux-ci puissent entreprendre des actions indépendantes, de mettre en œuvre des idées et de les mener à terme (Lumpkin et Dess, 1996).

Une étude effectuée par Weerawardena et Mort (2006, p. 33) démontre que l'orientation entrepreneuriale est liée à cette conceptualisation d'un « construit multidimensionnel » ayant comme dimension l'ouverture envers l'innovation, la gestion des risques et un comportement proactif. Lumpkin et Dess (1996) soutiennent en outre que l'ES favorise aussi l'autonomie des acteurs organisationnels visant à atteindre une mission sociale et que les acteurs sont invités à participer et à collaborer à la recherche de nouvelles initiatives.

La recherche d'occasions et le succès de leur exploitation nécessitent un ensemble d'orientations organisationnelles. Ces dernières doivent aussi s'orienter vers le marché. L'orientation vers le marché (OM) désigne "la génération, à l'échelle de l'organisation, d'information sur le marché, la diffusion de cette information au sein des différents services et la réaction à cette information à l'échelle de l'entreprise " (Jaworski et Kohli, 1993, p. 53). Cette orientation influence la manière dont l'information sur le marché est recueillie, partagée et utilisée au sein de l'entreprise.

Trois aspects sont fondamentaux à une orientation plus marchande, soit : la génération de renseignements en rapport au marché (de connaissances sur ce marché), la diffusion de ces renseignements au sein de 


\section{Hervieux, Fallu, \& Turcotte (2016)}

l'organisation et enfin la réactivité par rapport à ceux-ci (Jaworski et Kohli, 1993). «Générer des renseignements " consiste à recueillir des informations liées au marché (sur la clientèle, l'environnement concurrentiel et la réglementation) et à diffuser celles-ci (c'est-à-dire à les partager au sein de l'organisation). La réactivité implique l'utilisation des renseignements dans la réalisation des activités commerciales de l'organisation (Chen et Hsu, 2013) au niveau de la conception des produits, des processus ou de l'offre de services.

L'OE et l'OM sont des orientations complémentaires et lorsqu'on mesure l'OE, on doit mesurer également les autres orientations organisationnelles telles que l'OM. Ainsi, alors que l'orientation entrepreneuriale vise à encourager les comportements proactifs d'innovation et de prise de risque en laissant aux acteurs dans l'organisation l'autonomie nécessaire, l'orientation marché vise à encourager la collecte, la diffusion et l'utilisation d'informations liées au marché de l'organisation.

\section{HYBRIDITE, ORIENTATION ENTREPRENEURIALE ET ORIENTATION VERS LE MARCHE}

Les organisations d'ES sont considérées comme des organisations novatrices et entrepreneuriales (Lumpkin, Moss, Gras, Kato, et Amezcua, 2013). Elles ont les capacités dynamiques nécessaires à la survie d'une organisation hybride (Smith et Lewis, 2011). L'OE procure une souplesse stratégique permettant les actions autonomes et de prise de risque nécessaires à leur survie dans un milieu en évolution (Florin et Schmidt, 2011). Pour être performantes, ces organisations doivent également s'orienter vers le marché. Les objectifs de création simultanée d'une valeur publique et privée nécessitent une souplesse stratégique qu'apportent les orientations stratégiques entrepreneuriale et marchande (Markides, 2008; Smith et al., 2010; Florin et Schmidt, 2011).

Les organisations d'ES doivent aussi comprendre leur environnement. Or, dans l'entrepreneuriat social, c'est l'orientation vers le marché $(\mathrm{OM})$ qui permet de comprendre l'environnement. Des recherches antérieures ont signalé que les organisations d'ES tendent à adopter une orientation vers le marché (Dart, 2004; Nicholls, 2006). Celle-ci les aiderait à mieux comprendre leur environnement, ce qui peut à son tour favoriser l'innovation.

L'autonomie constitue un autre mécanisme important pour donner suite à l'innovation et aux idées entrepreneuriales. Le fait d'encourager les personnes à agir de façon autonome au sein de l'entreprise apporte la souplesse stratégique nécessaire, étant donné que :

Les nouvelles entreprises émanent souvent des niveaux inférieurs de l'organisation (Bower, 1970) et reflètent l'importance de l'autonomie pour les membres de l'organisation qui pourraient se trouver dans le contexte d'une initiative d'entreprise interne [...]; la liberté d'agir en toute indépendance constitue une dimension cruciale de l'orientation entrepreneuriale (Lumpkin et Dess, 1996, p. 141).

Ces orientations stratégiques sont pertinentes aussi pour les organisations hybrides durables qui entreprendront des activités commerciales au même titre que les entreprises à but lucratif tout en intégrant des préoccupations sociales et environnementales.

Étant donné que certaines innovations de produits et de services ou innovations organisationnelles peuvent avoir un effet et un succès limités lorsque la conjoncture du marché est très défavorable, les activités entrepreneuriales qui visent le développement 


\section{Hervieux, Fallu, \& Turcotte (2016)}

durable doivent non seulement tenir compte des difficultés du marché, mais aussi s'efforcer d'influer sur la conjoncture du marché (Schaltegger et Wagner, 2011, p. 224).

Par conséquent, l'OM influence l'OE en définissant les besoins et les attentes du marché avant de mettre au point des produits et des services novateurs. Mais en même temps, l'OE influence l'OM puisqu'elle oriente le type d'information recherchée par la direction. Elle donne également une impulsion aux activités d'OM. L'influence des dimensions de l'OE aura aussi un impact sur la façon dont les débouchés sur le marché seront exploités, et puisque l'entrepreneuriat favorise l'innovation, la proactivité et la prise de risque, « les entreprises qui présentent un niveau élevé d'activité entrepreneuriale adopteront des approches plus avancées à l'égard de l'environnement naturel » (Menguc et Ozanne, 2005, p. 433). Ces entreprises durables peuvent également connaître un succès commercial puisque plusieurs prétendent que la réduction des impacts sur l'environnement peut créer un avantage concurrentiel (Chen, 2008), augmenter leur part de marché (Porter et van der Lind, 1995) et, de manière générale, créer une image positive auprès des parties prenantes (Dibrell et al., 2011), puisque les « entreprises solidement orientées vers le marché sont proches de leur clientèle et en mesure de répondre à diverses exigences en constante évolution, que ce soit à l'égard des produits physiques ou des nombreux services associés à ces produits » (Dibrell et al., 2011, p. 470).

On constate donc que l'OE et l'OM constituent des orientations stratégiques importantes pour les organisations hybrides, dans la mesure où elles leur apportent les mécanismes nécessaires pour orienter les processus de décision. Étant donné que les organisations hybrides ont, par nature, des objectifs contradictoires, les deux orientations présentées ici constituent des capacités dynamiques orientant la prise de décision dans des situations complexes et ambiguës (Smith et Lewis, 2011).

\section{L 'IMPORTANCE DES RESSOURCES POUR LES ORGANISATIONS HYBRIDES}

Un autre aspect est lié au fait que les organisations hybrides d'entrepreneuriat social et d'entrepreneuriat durable sont généralement des PME (Bacq et al., 2013). Or, l'acquisition de ressources suffisantes est un aspect important pour les PME orientées vers le développement durable. Le manque de ressources financières est souvent identifié comme une barrière importante à l'introduction d'actions de responsabilité sociale et environnementale (RSE) dans les PME (Apospori, 2012). Les PME hybrides, sociales et durables qui visent un double ou triple objectif seront donc elles aussi touchées par la disponibilité des ressources financières.

Une façon de contourner cette faiblesse serait d'utiliser les réseaux qui sont des sources de capital social. En fait, les réseaux sont une source importante de ressources pour les PME et sont perçus comme des catalyseurs pour l'adoption et l'application de la RSE par les PME (Battaglia et al., 2010). Les organisations de l'ES ont à faire face à ces contraintes puisque l'environnement des organisations à vocation sociale est en changement, que les fonds gouvernementaux disponibles sont en baisse et que le nombre d'organisations cherchant à les obtenir est en hausse (Brouard et al., 2010). Afin de contrer ces difficultés, les organisations d'ES se tournent vers des ressources communautaires plutôt que de se concentrer sur l'accumulation de ressources à l'interne (Gras et Lumpkin 2012).

Les ressources communautaires sont accessibles dans les réseaux organisationnels et forment ce qui est communément appelé le capital social. Le capital social se compose des actifs présents dans les relations et serait lié aux réseaux sociaux de l'organisation. II inclut aussi les ressources disponibles à travers ces réseaux. Plus précisément, le « capital social désigne la capacité des acteurs à extraire les avantages de leurs structures sociales, de leurs réseaux et de leurs appartenances » (Davidsson \& Honig, 2003, p. 307). II facilite la découverte de bonnes occasions et permet que les ressources limitées soient diffusées à travers le réseau 


\section{Hervieux, Fallu, \& Turcotte (2016)}

(Davidsson \& Honig, 2003). Ainsi, en s'intégrant dans des réseaux organisationnels, les PME acquièrent des ressources qu'elles ne peuvent se permettre à l'interne. Voilà pourquoi nous avons également choisi de mesurer les différentes ressources dont disposent les organisations étudiées.

\section{METHODOLOGIE}

Les données ont été recueillies à l'automne 2012 au moyen d'un questionnaire autoadministré rempli en ligne. L'échantillon devait comprendre des organisations sociales et commerciales hybrides de formes juridiques variées, des organisations d'entrepreneuriat social ainsi que des PME commerciales. Les organisations d'entrepreneuriat social ont été sélectionnées au moyen de listes du Centre for Social Innovation, du Conseil pour les entreprises sociales du Canada et d'Ashoka et ClearlySo. Les PME commerciales proviennent du répertoire d'entreprises d'Industrie Canada. Nous avons reçu 244 réponses (114 organisations d'entrepreneuriat social, $130 \mathrm{PME}$ commerciales). Les répondants étaient les présidents-directeurs généraux ou les principaux informateurs de l'organisation.

Une analyse de regroupement a été réalisée afin d'identifier et de regrouper les PME selon la prise en compte des trois axes du développement durable (social, commercial et environnemental) dans les activités de développement et l'offre de produits. L'analyse de regroupement repose également sur ces trois axes. Les types de PME identifiés ont ensuite été profilés et comparés quant à leurs caractéristiques structurelles, leur degré d'actualisation des orientations entrepreneuriales et commerciales, et enfin, leurs ressources.

L'analyse de regroupement a permis d'identifier des grappes (clusters) d'organisations similaires sur la base d'une configuration de variables définitionnelles multiples (Gruber et al., 2010; Ketchen et Shook, 1996). Cette analyse a été effectuée en deux étapes (Punj et Stewart, 1983). À la première étape, les variables d'orientation sociale, commerciale et environnementale ont été soumises à une analyse de regroupement selon la méthode de Ward. À la seconde étape, les points médians (centroïdes) des regroupements identifiés à partir de la méthode de Ward ont été utilisés comme valeurs initiales dans une nouvelle analyse suivant la méthode par algorithme des centres de groupes non hiérarchique (K-means). La méthode de Ward agglomère hiérarchiquement les observations une à la fois dans un nombre prédéterminé de groupes, de manière à minimiser la variance entre les observations classées à l'intérieur de chacun des groupes. Les solutions produites par l'analyse ont ensuite été étudiées par rapport à la signification théorique des groupes au regard des variables analysées et de l'importance statistique des groupes. Trois groupes ont été retenus à l'issue de l'analyse de Ward. La méthode de Ward, parce qu'elle est agglomérative et hiérarchique, est sous-optimale dans la réaffectation des observations dans les groupes au fur et à mesure que l'analyse progresse. La méthode par algorithme des centres de groupes non hiérarchique a été utilisée afin d'optimiser la solution obtenue avec la méthode de Ward. Elle permet aussi de garantir un maximum d'homogénéité dans les groupes et un maximum d'hétérogénéité entre les groupes (Ketchen et Shook, 1996; Punj et Stewart, 1983). Cette méthode réattribue les observations itérativement entre les groupes jusqu'à ce qu'aucune modification significative ne soit observée dans les centres de groupes (c'est-à-dire qu'aucune observation ne soit réaffectée à un autre groupe). La stabilité de la solution retenue a été vérifiée à l'aide d'une validation croisée empirique. Nous avons effectué l'analyse de regroupement sur deux sous-échantillons sélectionnés au hasard (la moitié de l'échantillon au total) et comparé les résultats. Ces analyses ont présenté des résultats stables.

Toutes les mesures utilisées pour les analyses ont été adaptées à partir d'échelles validées dans le cadre de recherches antérieures et présentent de bonnes qualités psychométriques (fiabilité, validité convergente et discriminante) à l'issue des analyses factorielles exploratoires ainsi que des analyses factorielles confirmatoires effectuées. Spécifiquement, pour l'analyse de regroupement, les axes du développement durable (DD) ont été 
mesurés à partir de l'échelle de l'orientation sociale de Quarter et al. (2001) (Alpha $=0,91)$ et de l'échelle d'orientation environnementale de Banerjee et al. (2003) (Alpha $=0,96)$. L'orientation commerciale a été mesurée à partir d'une échelle de somme constante (maximum $=100 \%$ ) établissant le rapport en pourcentage entre la poursuite par l'organisation d'objectifs commerciaux et d'objectifs sociaux. L'OE a été mesurée à l'aide des trois mesures du modèle multidimensionnel de l'OE de Hughes et Morgan (2007; Lumpkin et Dess, 1996). La mesure de l'autonomie adoptée est celle de Lumpkin et Dess (2009) (Alpha : innovation $=0,89$; proactivité = $0,77$; risque $=0,88$; et autonomie $=0,89)$. Enfin, l'orientation marché $(\mathrm{OM})$ a été mesurée avec l'échelle MARKOR de Kohli, Jaworski et Kumar $(1993$, Alpha $=0,92)$. Cette échelle mesure les comportements et les routines de l'organisation de son OM (Zahra et al., 2008).

\section{RESULTATS}

Le tableau 1 présente les résultats descriptifs pour les variables ayant servi à classifier les PME. Dans chacun des regroupements, les PME intègrent différemment les dimensions du RSE. Le groupe des PME hybrides sociales est composé d'organisations qui sont essentiellement préoccupées par leurs objectifs sociaux et s'intéressent peu aux objectifs commerciaux. Leurs préoccupations à l'égard des objectifs environnementaux ne diffèrent pas de manière significative des préoccupations environnementales des PME commerciales. Les PME commerciales favorisent en premier lieu leurs objectifs commerciaux et, bien qu'elles se soucient également des objectifs de $\mathrm{DD}$, ces préoccupations ne sont clairement pas aussi importantes pour elles que les activités commerciales. Seules les PME hybrides durables intègrent simultanément les trois objectifs de la DD et favorisent fortement les préoccupations sociales et environnementales tout en poursuivant des objectifs commerciaux.

\section{Tableau 1 : Résultats descriptifs pour les variables utilisées pour classifier les PME}

\begin{tabular}{|c|c|c|c|c|c|}
\hline & & $\begin{array}{c}\text { Proportion } \\
\text { d'objectifs } \\
\text { commerciaux } \\
\text { (max. } 100)\end{array}$ & $\begin{array}{c}\text { Orientation sociale } \\
(\text { max. }=7)\end{array}$ & $\begin{array}{c}\text { Orientation } \\
\text { environnementale } \\
\text { (max. }=7)\end{array}$ \\
\hline $\mathbf{1}$ & $\begin{array}{c}\text { PME } \\
\text { sociales }\end{array}$ & $42(17,21)$ & $18,05^{2,3}(13,70)$ & $5,94^{3}(1,36)$ & $3,57(1,50)$ \\
\hline $\mathbf{2}$ & $\begin{array}{c}\text { PME hybrides } \\
\text { durables }\end{array}$ & $111(45,49)$ & $49,06^{3}(18,11)$ & $6,07^{3}(0,97)$ & $6,26^{1,3}(0,78)$ \\
\hline $\mathbf{3}$ & $\begin{array}{c}\text { PME } \\
\text { commerciales }\end{array}$ & $91(37,30)$ & $79,81(13,60)$ & $3,21(1,31)$ & $3,73(1,70)$ \\
\hline & Total & $\mathbf{2 4 4}$ & $\mathbf{5 5 , 1 9 ( 2 7 , 0 4 )}$ & $\mathbf{4 , 9 8}(\mathbf{1}, 80)$ & $\mathbf{4 , 8 5}(1,84)$ \\
\hline
\end{tabular}

Les chiffres, en exposant 1 (PME hybrides sociales), 2 (PME hybrides durables) et 3 (PME commerciales), indiquent des écarts statistiquement significatifs entre les moyennes $(p<0,001)$.

L'âge, la taille et le chiffre d'affaires des organisations jouent un rôle déterminant pour expliquer les orientations stratégiques des PME (Covin et Slevin, 1991). Avant d'analyser les variables liées à l'orientation stratégique, nous nous sommes assurés que les regroupements ne varient pas de façon significative en fonction de ces caractéristiques organisationnelles. Comme l'indique le tableau 2, les PME sociales, hybrides et commerciales ne présentent pas de différences significatives d'âge $(F(2,243)=2,35 ; p=0,10)$, de nombre 
d'employés $(F(2,243)=0,19 ; p=0,83)$ ou de chiffre d'affaires $\left(\chi^{2}(4)=7,89 ; p=0,10\right)$. Les différences observées dans les orientations stratégiques privilégiées par l'équipe de direction des PME ne se rapportent donc pas à leurs caractéristiques structurelles, mais plutôt à leur identité définie en fonction des axes du DD.

Tableau 2 : Activités des organisations en fonction des groupes

\begin{tabular}{|c|c|c|c|c|}
\hline & PME sociales & $\begin{array}{c}\text { PME hybrides } \\
\text { durables }\end{array}$ & $\begin{array}{c}\text { PME } \\
\text { commerciales }\end{array}$ & Total \\
\hline $\begin{array}{c}\text { Âge } \\
\text { Moyenne (É-T) }\end{array}$ & $20,21(14,39)$ & $14,79(14,56)$ & $16,75(13,05)$ & $16,45(14,06)$ \\
\hline $\begin{array}{c}\text { Taille (employés) } \\
\text { Moyenne (É-T) }\end{array}$ & $22,50(43,36)$ & $27,61(60,05)$ & $28,62(53,0)$ & $27,11(54,70)$ \\
\hline \multicolumn{4}{|c|}{ Chiffre d'affaires \% } \\
\hline Inférieur à 250 000 & 45,00 & 42,99 & 27,06 & 37,50 \\
\hline 250 000 à 2 millions & 35,00 & 39,25 & 42,35 & 39,66 \\
\hline Plus de 2 millions & 20,00 & 17,76 & 30,59 & 22,84 \\
\hline
\end{tabular}

\section{PROFIL DES PME SELON LEUR GROUPE D'APPARTENANCE}

Nous avons ensuite établi le profil des orientations stratégiques adoptées par les PME et des ressources dont celles-ci disposent.

\section{ORIENTATIONS STRATEGIQUES ET TYPES DE PME}

Les PME diffèrent de façon importante en ce qui a trait aux dimensions de l'orientation entrepreneuriale et de l'orientation vers le marché adoptées. Le tableau 3 montre aussi que les PME du groupe des organisations hybrides durables ont des scores nettement supérieurs au titre des capacités stratégiques dynamiques comparativement à ceux des PME commerciales et sociales. Rappelons que les organisations hybrides durables diffèrent significativement des PME sociales et commerciales mais diffèrent peu quant à leurs orientations stratégiques dynamiques (tableau 3).

Enfin, les organisations d'entrepreneuriat social qui ne font pas partie du groupe d'organisations hybrides durables adoptent des orientations stratégiques similaires à celles des organisations d'entrepreneuriat commercial. Elles adoptent aussi bien les modes organisationnels que les façons de traiter en affaires des organisations commerciales à but lucratif. Les objectifs de ces organisations diffèrent cependant en matière d'objectifs sociaux et commerciaux, accordant en effet la priorité aux objectifs sociaux plutôt qu'aux objectifs commerciaux. 
Hervieux, Fallu, \& Turcotte (2016)

Tableau 3 : Comparaison des orientations stratégiques des PME

\begin{tabular}{|c|c|c|c|c|c|c|c|}
\hline & \multicolumn{4}{|c|}{$\begin{array}{c}\text { Statistiques descriptives } \\
\text { Moyennes (É-T) }\end{array}$} & \multicolumn{3}{|c|}{$\begin{array}{l}\text { Test t pour échantillons } \\
\text { indépendants }\end{array}$} \\
\hline & $\begin{array}{l}\text { PME } \\
\text { sociales }\end{array}$ & $\begin{array}{c}\text { PME } \\
\text { hybrides } \\
\text { durables }\end{array}$ & $\begin{array}{l}\text { PME } \\
\text { commer. }\end{array}$ & Total & $\begin{array}{l}\text { Hybrides } \\
\text { vs. } \\
\text { sociales }\end{array}$ & $\begin{array}{l}\text { Hybrides } \\
\text { vs. } \\
\text { commer. }\end{array}$ & $\begin{array}{l}\text { Sociales } \\
\text { vs. } \\
\text { commer. }\end{array}$ \\
\hline $\mathrm{OE}$ & & & & & $\mathrm{ddl}=151$ & $\mathrm{dd}=200$ & $\mathrm{ddl}=131$ \\
\hline Innov. & $\begin{array}{c}5,60 \\
(1,33)\end{array}$ & $\begin{array}{c}6,29 \\
(0,78)\end{array}$ & $\begin{array}{c}5,49 \\
(1,24)\end{array}$ & $\begin{array}{c}5,87 \\
(1,13)\end{array}$ & $3,94^{* * *}$ & $5,60^{\star \star *}$ & 0,49 \\
\hline Proac. & $\begin{array}{c}4,49 \\
(1,51)\end{array}$ & $\begin{array}{c}5,08 \\
(1,22)\end{array}$ & $\begin{array}{c}4,67 \\
(1,22)\end{array}$ & $\begin{array}{c}4,83 \\
(1,29)\end{array}$ & $2,50^{*}$ & $2,35^{*}$ & $-0,76$ \\
\hline Risque & $\begin{array}{c}4,83 \\
(1,56)\end{array}$ & $\begin{array}{c}5,29 \\
(1,23)\end{array}$ & $\begin{array}{c}4,89 \\
(1,26)\end{array}$ & $\begin{array}{c}5,06 \\
(1,31)\end{array}$ & $1,92 \dagger$ & $2,26^{*}$ & $-0,26$ \\
\hline Auton. & $\begin{array}{c}5,38 \\
(1,09)\end{array}$ & $\begin{array}{c}5,93 \\
(0,78)\end{array}$ & $\begin{array}{c}5,09 \\
(1,29)\end{array}$ & $\begin{array}{c}5,52 \\
(1,12)\end{array}$ & $3,47^{* *}$ & $5,72^{\star \star *}$ & 1,28 \\
\hline OM & $\begin{array}{c}5,08 \\
(1,08)\end{array}$ & $\begin{array}{c}5,64 \\
(0,93)\end{array}$ & $\begin{array}{c}5,24 \\
(1,08)\end{array}$ & $\begin{array}{c}5,40 \\
(1,04)\end{array}$ & $3,18^{* *}$ & $2,77^{\star *}$ & $-0,83$ \\
\hline
\end{tabular}

${ }^{* * *} p<0,001 ;{ }^{* *} p<0,01 ;{ }^{*} p<0,05 ; \uparrow p<0,06$. Tous les tests sont bilatéraux.

\section{TYPES DE RESSOURCES EN FONCTION DES REGROUPEMENTS DE PME}

Nous avons également étudié comment ces organisations diffèrent en fonction des ressources dont elles disposent. II s'agit :

- Des ressources en capital social, c'est-à-dire des actifs tangibles et intangibles présents dans les réseaux et les relations;

- Des ressources organisationnelles, c'est-à-dire des compétences, méthodes et capacités organisationnelles telles « la capacité à livrer de nouveaux produits à un marché dans un délai raisonnable » (Covin \& Slevin, 1991, p. 15);

- Des ressources humaines, c'est-à-dire des capitaux humains, compétences et connaissances de la direction et des employés (Coleman 1988);

- Des ressources financières, c'est-à-dire des capitaux financiers disponibles pour l'organisation.

Les PME que nous avons étudiées diffèrent peu en ce qui concerne leurs niveaux de ressources financières, humaines et organisationnelles, mais diffèrent davantage en matière de capital social (tableau 4). Les organisations hybrides durables et les organisations d'entrepreneuriat social présentent des niveaux supérieurs de capital social, en particulier les organisations hybrides durables. En effet, les organisations qui poursuivent des objectifs sociaux, dont les organisations d'entrepreneuriat social et les organisations hybrides durables, s'efforcent également de travailler en étroite collaboration avec la communauté (Hervieux et Turcotte, 2010). Tandis que les « entrepreneurs (autant les entrepreneurs commerciaux que sociaux) sont (plutôt) intégrés au sein d'un important contexte social auquel elles réagissent activement » (Chamlee-Wright, 2008, p. 46). Les organisations d'entrepreneuriat social diffèrent des entrepreneurs commerciaux dans la mesure où elles ont «tendance à utiliser les ressources de manière coopérative et souvent à les partager avec d'autres organisations » (Dacin, Dacin et Matear, 2010, p. 49), ce qui favorise à son tour la création de capital social. 
Hervieux, Fallu, \& Turcotte (2016)

Tableau 4 : Ressources et types de PME

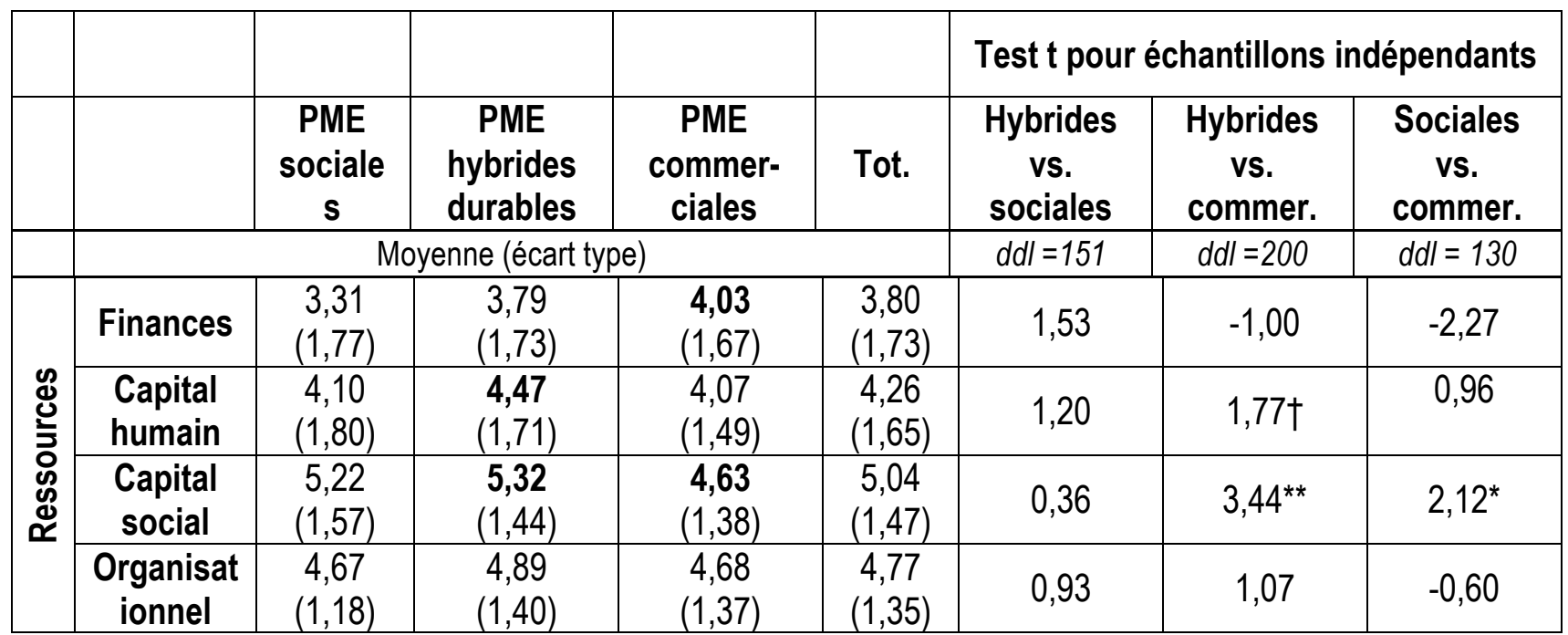

${ }^{* * *} p<0,001 ;{ }^{* *} p<0,01 ;{ }^{*} p<0,05 ; \uparrow p<0,10$ (tests bilatéraux)

\section{CONCLUSION}

La structure du développement durable $(\mathrm{DD})$ dans les PME durables révèle la présence d'un groupe d'organisations qui équilibrent leur recherche des trois objectifs en s'assurant que leurs objectifs commerciaux ne dominent pas leurs objectifs sociaux et environnementaux. Le niveau des objectifs commerciaux recherché par les organisations hybrides diffère de celui des PME d'entrepreneuriat social et des PME commerciales. Pourtant, les objectifs sociaux des PME d'entrepreneuriat durables ne diffèrent pas des objectifs des PME d'entrepreneuriat social. Ces organisations hybrides durables et sociales présentent des niveaux d'objectifs sociaux supérieurs à ceux des PME commerciales. Seules les organisations hybrides durables présentent aussi des niveaux élevés de préoccupations environnementales.

Les organisations d'entrepreneuriat social ne peuvent pas toutes être considérées comme hybrides étant donné que plusieurs se concentrent sur des objectifs sociaux et s'intéressent peu aux questions commerciales et environnementales. Les organisations hybrides d'entrepreneuriat social sont également des organisations hybrides durables dans la mesure où elles intègrent les trois dimensions du DD. Par ailleurs, nous notons également que les organisations hybrides durables ne sont pas toutes des organisations d'entrepreneuriat social, ou ne sont pas encore reconnues comme telles, puisqu'un grand nombre d'entre elles appartiennent à notre échantillon de PME commerciales.

Les organisations hybrides durables sont dans l'ensemble plus axées sur l'entrepreneuriat que toutes les autres, car elles sont les plus fortement orientées vers l'innovation, la prise de risque, la proactivité et l'autonomie. Elles sont également plus orientées vers le marché que toutes les autres organisations et font face aux tensions créées par l'intégration de nombreux objectifs opposés car elles ont adopté une orientation stratégique dynamique dans laquelle les actions entrepreneuriales sont guidées par la connaissance du marché. 


\section{Hervieux, Fallu, \& Turcotte (2016)}

Les organisations hybrides durables sont des organisations pluralistes qui acceptent les tensions inhérentes liées aux objectifs du développement durable. Elles acceptent aussi les tensions liées au triple objectif, ainsi que celles liées à leurs orientations stratégiques. Les organisations hybrides sont le plus fortement orientées vers le marché et s'efforcent ainsi de comprendre son évolution.

Dans la mesure où elles adoptent également une orientation entrepreneuriale solide, les organisations hybrides semblent agir de manière plus proactive que les autres PME et par la même occasion privilégier l'innovation et la prise de risque. Ceci est à son tour rendu possible parce qu'elles accordent aux membres de l'organisation l'autonomie nécessaire à la prise de risque. Par conséquent, même si l'adoption des triples résultats peut être conçue comme une source de tensions pour l'organisation, les réponses mises en place par les organisations hybrides durables pourraient être interprétées comme un effort en vue de travailler avec ces tensions par le biais de liens avec le marché et en conservant une longueur d'avance sur leurs concurrents.

\section{REFERENCES}

Albert, S., \& Whetten, D. A. (1985). Organizational identity. Research in Organizational Behavior, 7, 263-295.

Apospori, E ; Zografos, K. G. \& Magrizos, S. (2012). SME corporate social responsibility and competitiveness: A literature review. International Journal of Technology Management, 58(1/2), 10-31.

Atuahene-Gima, \& Ko, K. (2001). An empirical investigation of the effect of market orientation alignment on product innovation. Organization Science, 12(1), 54-74.

Austin, J., Stevenson, H., \& Wei-Skillern, J. (2006). Social and commercial entrepreneurship: The same, different or both? Entrepreneurship, Theory and Practice, 30(1), 1-22.

Bacq, S., Hartog., C., \& Hoogendoorn, B. (2013). A quantitative comparison of social and commercial entrepreneurship: Toward a more nuanced understanding of social entrepreneurship organizations in context. Journal of Social Entrepreneurship, 4(1), 40-68.

Baker, W.E., \& Sinkula, J. M. (2009). The complementary effects of market orientation and entrepreneurial orientation on profitability in small businesses. Journal of Small Business Management, 47(4), 443-464.

Banerjee, S.B., Iyer, E.S., \& Kashyap, R.K. (2003). Corporate environmentalism: Antecedents and influence of industry type. Journal of Marketing, 67(2), 106-122.

Battaglia, M., Bianchi, L., Frey, M. \& Iraldo, F. (2010). An innovative model to promote CSR among SMEs operating in industrial clusters: Evidence from an EU project. Corporate Social Responsibility and Environmental Management, 17(3), 133-141.

Biondi, V., Iraldo, F., \& Meredith, S. (2002). Achieving sustainability through environmental innovation: The role of SMEs. International Journal of Technology Management, 24(5), 612-626.

Battilana, J., Lee, M., Walker, J., \& Dorsey, C. (2012). In search of the hybrid ideal. Stanford Social Innovation Review, Summer, 49-55. URL : http://ssir.org/articles/entry/in_search_of_the_hybrid_ideal [May 3, 2016].

Bhuian, S., Menguc, B., \& Bell, S. (2005). Just entrepreneurial enough: The moderating effect of entrepreneurship on the relationship between market orientation and performance. Journal of Business Research, 58(1), 9-17.

Brouard, F., et Larivet, S. (2010). Essay of clarifications and definitions of related concepts of social enterprise, social entrepreneur and social entrepreneurship. Dans A. Fayolle \& H. Matlay (dir.), Handbook of Research in Social Entrepreneurship. Northampton, MA: Edward Elgar Publishing.

Brouard, F., Larivet, S., \& Sakka, O. (2010). Entrepreneuriat social et participation citoyenne. Revue canadienne de recherche sur les OSBL et l'économie sociale, automne, 46-64.

Chamlee-Wright, E. (2008). The structure of social capital: An Austrian perspective on its nature and development. Review of Political Economy, 20(1), 41-58. 


\section{Hervieux, Fallu, \& Turcotte (2016)}

Chen, Y. S. (2008). The positive effect of green intellectual capital on competitive advantages of firms. Journal of Business Ethics, 77(3), 271-286.

Cohen, B., \& Winn, M.I. (2007). Market imperfections, opportunity and sustainable entrepreneurship. Journal of Business Venturing, 22(1), 29-49.

Covin, J.G., and Slevin, D.P. (1991). A conceptual model of entrepreneurship as firm behavior. Entrepreneurship Theory and Practice, 16(1), 7-25.

Covin, J.G., \& Wales, W.J. (2012). The measurement of entrepreneurial orientation. Entrepreneurship Theory and Practice, 36(4), 677-702.

Dacin, P.A., Dacin, M.T., \& Matear, M. (2010). Social entrepreneurship: Why we don't need a new theory and how we move forward from here. Academy of Management Perspectives, 24(3), 37-57.

Dart, R. (2004). Charities in business, business in charities, charities and business-mapping and understanding the complex nonprofit/business interface. The Philanthropist, 18(3), 181-199.

Dees, J.G. (1998). The meaning of "social entrepreneurship." Duke Fuqua School of Business. URL : https://entrepreneurship.duke.edu/news-item/the-meaning-of-social-entrepreneurship/ [May 3, 2016].

Dibrell, C., Craig, J., \& Hansen, E. (2011). Natural environment, market orientation, and firm innovativeness: An organizational life cycle perspective. Journal of Small Business Management, 49(3), 467-489.

Eikenberry, A.M., \& Kluver, J.D. (2004). The marketization of the nonprofit sector: Civil society at risk? Public Administration Review, 64(2), 132-140.

Florin, J. \& Schmidt, E. (2011). Creating shared value in the hybrid venture arena: A business model innovation perspective. Journal of Social Entrepreneurship, 2(2), 165-197.

Gruber, M., Heinemann, F., Brettel, M., \& Hungeling, S. (2010). Configurations of resources and capabilities and their performance implications: An exploratory study on technology ventures. Strategic Management Journal, 31(12), 1337-1356.

Hervieux, C., Gedajlovic, E., \& Turcotte, M.-F. (2010). The legitimization of social entrepreneurship. Journal of Enterprising Communities: People and Places in the Global Economy, 4(1), 37-67.

Hervieux, C., \& Turcotte, M.-F. (2010). Social entrepreneurs' actions in networks. Dans A. Fayolle \& H. Matlay (dir.), Handbook of Research in Social Entrepreneurship. Northampton, MA: Edward Elgar Publishing.

Hughes, M., \& Morgan, R.E. (2007). Deconstructing the relationship between entrepreneurial orientation and business performance at the embryonic stage of firm growth. Industrial Marketing Management, 36(5), $651-661$.

Jaworski, B.J., \& Kohli, A.K. (1993). Market orientation: Antecedents and consequences. Journal of Marketing, $57(3), 53-70$.

Ketchen Jr., D.J., \& Shook, C.L. (1996). The application of cluster analysis in strategic management research: An analysis and critique. Strategic Management Journal, 17(6), 441-459.

Kohli, A.K., \& Jaworski, B.J. (1990). Market orientation: The construct, research propositions, and managerial implications. Journal of Marketing, 54(2), 1-18.

Kohli, A.K., Jaworski, B.J., \& Kumar, A. (1993). MARKOR: A measure of market orientation. Journal of Marketing Research, 30(4), 467-477.

Larson, A. (2000). Sustainable innovation through an entrepreneurship lens. Business Strategy and the Environment, 9(5), 304-317.

Lasprogata, G.A., \& Cotton, M.N. (2003). Contemplating enterprise: The business and legal challenges of social entrepreneurship. American Business Law Journal, 41(1), 67-113.

Longo, M., Mura, M., \& Bonoli, A. (2005). Corporate social responsibility and corporate performance: The case of Italian SMEs. Corporate Governance, 5(4), 28-42.

Lumpkin, G.T., \& Dess, G.G. (1996). Clarifying the entrepreneurial orientation construct and linking it to performance. Academy of Management Review, 21(1), 135-172. 


\section{Hervieux, Fallu, \& Turcotte (2016)}

Lumpkin, G.T., Moss, T.W., Gras, D.M., Kato, S., Amezcua, S.K. (2013). Entrepreneurial processes in social contexts: How are they different, if at all? Small Business Economics, 40(3), 761-783.

Mair, J., \& Marti, I. (2006). Social entrepreneurship research: A source of explanation, prediction, and delight. Journal of World Business, 41(1), 36-44.

Matsuno, K., Mentzer, J.T., \& Özsomer, A. (2002). The effects of entrepreneurial proclivity and market orientation on business performance. Journal of Marketing, 66(3), 18-32.

Menguc, B., \&. Ozanne, L.K. (2005). Challenges of the "green imperative": A natural resource-based approach to the environmental orientation-business performance relationship, Journal of Business Research, $58(4), 430-438$.

Morris, M.H., Coombes, S., Schindehutte, M., \& Allen, J. (2007). Antecedents and outcomes of entrepreneurial and market orientations in a non-profit context: Theoretical and empirical insights. Journal of Leadership \& Organizational Studies, 13(4), 12-39.

Nicholls, A. (2006). Introduction: The meanings of social entrepreneurship. Dans A. Nicholls (dir.), Social entrepreneurship: New paradigms of sustainable social change (pp. 1-36). Oxford: Oxford University Press.

Nicholls, A., (2010). The legitimacy of social entrepreneurship: Reflexive isomorphism in a pre-paradigmatic field. Entrepreneurship: Theory and Practice, 34(4), 611-633.

Nicholls, A., \& Cho, A.H. (2006). Social entrepreneurship: The structuration of a field. Dans Nicholls, A. (dir.), Social Entrepreneurship: New Models of Sustainable Social Change (pp. 99-118). Oxford: Oxford University Press.

Peredo, A.M., \& McLean, M. (2006). Social entrepreneurship: A critical review of the concept. Journal of World Business, 41(1), 56-65.

Perrini, F., dir. (2006). The new social entrepreneurship: What awaits social entrepreneurial ventures? Northampton, MA: Edward Elgar Publishing.

Perrini, F., Russo, A., \& Tencati, A. (2007). CSR strategies of SMEs and large firms. Evidence from Italy, Journal of Business Ethics, 74(3), 285-300.

Porter, M.E., \& van der Lind, C. (1995). Green and competitive: Ending the stalemate. Harvard Business Review, 73(5), 120-134.

Pratt, M.G., \& Foreman, P.O. (2000). Classifying managerial responses to multiple organizational identities. Academy of Management Review, 25(1), 18-42.

Punj, G., \& Stewart, D.W. (1983). Cluster analysis in marketing research: Review and suggestions for application. Journal of Marketing Research, 20(2), 134-148.

Quarter, J., Sousa, J., Richmond, B.J., \& Carmichael, I. (2001). Comparing member-based organizations within a social economy framework. Nonprofit and Voluntary Sector Quarterly, 30(2), 351-375.

Ruebottom, T. (2013). The microstructures of rhetorical strategy in social entrepreneurship: Building legitimacy through heroes and villains. Journal of Business Venturing, 28(1), 98-116.

Schaltegger, S., \& Wagner, M. (2011). Sustainable entrepreneurship and sustainability innovation: Categories and interactions. Business Strategy and the Environment, 20(4), 222-237.

Shapero, A. (1975). The displaced, uncomfortable entrepreneur. Psychology Today, 7, 83-89.

Sharir, M., \& Lerner, M. (2006). Gauging the success of social ventures initiated by individual social entrepreneurs. Journal of World Business, 41(1), 6-20.

Smith, W.K., \& Lewis, M.W. (2011). Toward a theory of paradox: A dynamic equilibrium model of organizing. Academy of Management Review, 36(2), 381-483.

Spence, L., Guerib, J.B.B., \& Biwolé, V.O. (2011). Sustainable entrepreneurship: Is entrepreneurial will enough? A north-south comparison. Journal of Business Ethics, 99(3), 335-367.

Weerawardena, J., McDonald, R.E., \& Mort, G.S. (2010). Sustainability of nonprofit organizations: An empirical investigation. Journal of World Business, 45(4), 346-356. 
Wilson, F., \& Post, J.E. (2013). Business models for people, planet (\& profits): Exploring the phenomena of social business, a market-based approach to social value creation. Small Business Economics, 40(3), 715-737.

Winn, M.I., \& Kirchgeorg, M. (2005). The siesta is over: A rude awakening from sustainability myopia. Dans S. Sharma et M. Starik (dir.), Corporate Environmental Strategy and Competitive Advantage (pp. 232258). Northampton, MA: Edward Elgar Publishing.

Wolverton, B. (2003). Surviving tough times. The Chronicle of Philanthropy, October 30.

Zahra, S. A. (2008). Being entrepreneurial and market driven: Implications for company performance. Journal of Strategy and Management, 1(2), 125-142.

Zahra, S., Gedajlovic, E., Neubaum, D. O., \& Shulman, J. M. (2009). A typology of social entrepreneurs: Motives, search processes, and ethical challenges. Journal of Business Venturing, 24(5), 519-532.

\section{LES AUTEURS / ABOUT THE AUTHORS}

Chantal Hervieux is Assistant Professor at Saint Mary's-Sobey School of Busines. Email: chantal.hervieux@smu.ca

Jean-Mathieu Fallu est un candidat au doctorat à HEC Montréal-École des Hautes Études commerciales. Email : jean-mathieu.fallu@hec.ca

Marie-France Turcotte est professor titulaire Université du Québec à Montréal-École des sciences de la gestion. Email : turcotte.marie-france@uqam.ca 